\title{
EFEITOS DA DANÇATERAPIA E DA CINESIOTERAPIA SOBRE O EQUILÍBRIO, QUALIDADE DE VIDA, AGILIDADE E FLEXIBILIDADE DE IDOSOS ASILARES
}

\author{
Isabela Marie Komegae, Juliana Alves Martins, Siméia Gaspar Palácio e Kelley \\ Cristina Coelho \\ Unicesumar \\ isabelakomegae@hotmail.com
}

Acesso DOI: http://dx.doi.org/10.34059/ciejop.2019v27i1-10

\section{RESUMO}

O presente estudo foi realizado com idosos asilares na Associação de Amparo à Pessoas Idosas - Wajun Kai de Maringá após a aprovação do Comitê de Ética em Pesquisa da Unicesumar e da assinatura do Termo de Consentimento Livre e Esclarecido. Foram selecionados aleatoriamente 5 idosos saudáveis com idade variando entre 65-85 anos e excluídos do estudo indivíduos com doenças neurológicas, cadeirantes e que apresentavam déficit de compreensão. $\mathrm{Na}$ seqüência, os idosos foram avaliados em relação ao equilíbrio utilizando a Escala de Berg, flexibilidade através do teste sentar e alcançar com o banco de Wells, qualidade de vida pelo questionário SF-36 e agilidade por meio do teste Time Upand Go. Ao término da avaliação, foram realizadas as práticas de dançaterapia que constaram de 10 sessões, realizadas em grupo, com duração de 40 minutos, utilizando diferentes ritmos, alongamentos e relaxamentos musculares. Posteriormente, os participantes foram reavaliados utilizando os mesmos instrumentos para verificação dos resultados alcançados e os dados obtidos foram analisados de forma descritiva, sendo possível concluir que a dança associada a cinesioterapia contribuiu para o equilíbrio e a qualidade de vida dos idosos tratados.

PALAVRAS - CHAVE: geriatria, dança, qualidade de vida.

\section{ABSTRACT}

The present study was performed with elderly people in the Association of Shelter for the Elderly - Wajun Kai of Maringá after the approval of the Ethics Committee in Research of Unicesumar and the signing of the Term of Free and Informed Consent. Five healthy elderly individuals, aged 65-85 years, were randomly selected and excluded from the study were individuals with neurological diseases, wheelchair users, and those with comprehension deficits. In the sequel, the elderly were evaluated for balance using the Berg Scale, flexibility through the sit-and-reach test with the Wells bench, SF-36 quality of life, and agility using the Time Up and Go test. After completion of the evaluation, the dance practices were performed, which consisted of 10 sessions, performed in a group, lasting 40 minutes, using different rhythms, stretching and muscle relaxation. Subsequently, the participants were reassessed using the same instruments to verify the results achieved and the data obtained were analyzed in a descriptive way, and it was possible to conclude that the 
dance associated with kinesiotherapy contributed to the balance and the quality of life of the treated elderly.

KEYWORDS: geriatrics, dance, quality of life.

\section{INTRODUÇÃO}

Segundo dados do IBGE (2016), a expectativa de vida do brasileiro elevou-se em 30,3 anos. Esta ascensão está relacionada a fatores como campanhas de vacinação, incorporação de avanços da medicina no âmbito da saúde pública, atividade de agentes comunitários de saúde, entre outros. Aliado a este aumento significativo na expectativa de vida, ocorre de forma concomitante o aumento de comorbidades que afetam a população idosa. Estima-se que no ano de 2020, o Brasil terá aproximadamente 30,9 milhões de pessoas com idade superior a 60 anos (Moraes, 2008).

O envelhecimento por si só consiste em um processo natural na vida dos seres humanos no qual ocorrem alterações fisiológicas, sociais, comportamentais, morfológicas, entre outras. Dentre as alterações ocorridas na fase de senescência destaca-se como foco de investigação deste estudo as que acontecem ao nível osteomuscular, as quais se destacam a redução de massa corporal em decorrência da menor prática de atividade física, alimentação inapropriada e diminuição da taxa de água corpórea.

Outras alterações decorrentes da senescência: Redução muscular e flexibilidade, diminuição do peso corpóreo e maior risco no desenvolvimento de patologias crônicas. Tais variáveis quando ocorrem concomitantemente resultam em compensações na marcha e déficits no equilíbrio (Silva et al., 2008), além de conseqüente comprometimento na mobilidade das articulações. Conseqüentemente, o indivíduo idoso pode apresentar uma maior propensão à quedas e dificuldade na realização das atividades de vida diária (AVD's), apresentando comprometimentos em sua qualidade de vida (Reis et al., 2011).

Entre as principais mudanças ocorridas com o avançar da idade, destacamse a redução na massa corporal (sarcopenia) e na flexibilidade. Esta redução na flexibilidade pode ocasionar osteoartrite em decorrência do desgaste da cartilagem presente nas articulações (Francisco Netto, 2004). 
As alterações ocorridas em nível de tecido ósseo durante o envelhecimento advêm de três fatores principais: alterações nas taxas hormonais naturais da idade; nutrição deficiente e redução na prática de atividade física. A força realizada pelas alavancas ósseas provém dos minerais presentes nos ossos que com o avançar da idade, tornam-se mais frágeis, levando conseqüentemente ao maior risco de microfraturas (Spirduso, 2005).

Tendo em vista que as comorbidades estão diretamente relacionadas ao aumento da expectativa de vida da população, se faz necessário incluir a prática regular de atividade física visando uma melhor qualidade de vida e diminuição das possíveis patologias que possam acometer o idoso. A dança levou uma das modalidades de tratamento que proporciona benefícios nos aspectos psicológicos, motores, cognitivos, sociais e no equilíbrio corporal (Barbosa et al., 2013)

Deste modo, o presente estudo teve como objetivo verificar a efetividade da dançaterapia de idosos asilares com relação ao equilíbrio, flexibilidade, agilidade e qualidade de vida.

\section{METODOLOGIA}

A presente pesquisa foi do tipo intervencional e teve início após aprovação pelo Comitê de Ética e Pesquisa da Unicesumar sob o parecer 3.022.123. A mesma foi realizada na Associação de Amparo à Pessoas Idosas - Wajun Kai de Maringá com 5 idosos saudáveis e idade variando de 65 a 85 anos. Foram excluídos do estudo indivíduos portadores de doenças neurológicas, cadeirantes e com déficit de compreensão.

Após a assinatura do Termo de Consentimento Livre e esclarecido os pacientes foram submetidos a uma avaliação inicial em relação à flexibilidade, agilidade, equilíbrio e qualidade de vida.

$\mathrm{Na}$ avaliação da qualidade de vida foi utilizado o questionário SF-36 que avalia oito domínios, os quais consistem em aspectos físicos, capacidade funcional, estado geral de saúde, vitalidade, aspectos emocionais, dor, aspectos sociais e 
aspectos emocionais e saúde mental. O mesmo é pontuado de 0 a 100, sendo que zero corresponde ao pior estado de saúde e 100 o melhor (Ciconelli, 1999).

Para a variável flexibilidade foi utilizado o banco de Wells também conhecido como teste de sentar e alcançar, cujo objetivo é medir a amplitude do alongamento da parte posterior do tronco e membros inferiores. Para a execução do teste, o paciente foi orientado a sentar-se sobre um colchonete, realizar uma flexão do tronco sobre o quadril na máxima amplitude possível, mantendo os membros superiores estendidos à frente com uma mão colocada sobre a outra, deslizando os dedos ao longo da régua. O procedimento foi realizado três vezes, sendo considerada a medida de flexibilidade a maior distância atingida das três tentativas (Ribeiro et al.,2010).

A flexibilidade foi classificada utilizando como critérios os parâmetros da Canadian Standardized Test of Fitness (CTF), sendo classificada em excelente, muito boa, razoável e necessita melhorar (Fitness Canada, 1986).

O equilíbrio foi avaliado pela escala de Berg, cujos escores oscilam de 0 a 56 pontos, sendo que, quanto maior a pontuação, mais eficiente é o equilíbrio do sujeito. A escala possui itens onde cada um deles apresenta uma escala ordinal com 5 opções que variam de 0 a 4 pontos. Escores de 56 a 54 serão proporcionais a um aumento de 3 a 4\% no risco de quedas; entre 54 e 46, apresentam uma relação de aumento de 6 a $8 \%$ e abaixo de 36 pontos associam-se risco de quedas de praticamente 100\% (Silva et al., 2008; Scheicher; Pimentel, 2009).

Para avaliar a agilidade foi utilizado o teste Time Up and Go que verifica a probabilidade de quedas, o equilíbrio dinâmico e a capacidade de deslocamento. Para realizar o teste, foi solicitado que o indivíduo se levantasse de uma cadeira,em seguida, percorresse uma distância de 3 metros, realizasse uma volta e retornasse ao ponto inicial. $O$ teste foi realizado três vezes, sendo adotada como referência a mensuração de menor tempo. Pessoas que demandam mais de 30 segundos para o percurso do teste necessitam de maior auxílio em suas atividades de vida diária, assim como na mobilidade, apresentando um risco maior de quedas. Os indivíduos que realizam o teste com 20 segundos ou menos possuem a necessidade de ajuda na transferência. Em contrapartida, aqueles que cumprem o teste em menos de 10 
segundos não necessitam de auxílio e não apresentam déficit no equilíbrio (Lopes et al., 2009).

Ao término da avaliação, foi iniciado o atendimento fisioterapêutico que constou de 10 sessões utilizando a dançaterapia, uma vez por semana com duração de 40 minutos, utilizando diferentes ritmos musicais, precedidos e finalizados com alongamentos ativos dos membros inferiores, superiores e da musculatura do tronco, além de relaxamento.

Ao término dos atendimentos, os pacientes foram reavaliados utilizando os mesmos instrumentos para verificação dos resultados alcançados.

\section{RESULTADOS}

No estudo foram selecionados 5 idosos, sendo a amostra composta por $60 \%$ de mulheres e 40\% por homens, com uma média de idade de 76 anos. No referente ao Teste de Sentar e Alcançar (Banco de Wells) foi utilizado como parâmetro os critérios da Canadian Standardized Test of Fitness (CSTF) que classifica os resultados da flexibilidade, de acordo com o gênero e a faixa etária em excelente, muito bom, bom, razoável e necessita melhorar necessita melhorar (Tabela 01).

No que diz respeito à flexibilidade a maioria dos pacientes se enquadraram na classificação "necessita melhorar", com exceção de uma idosa que se enquadrava na classificação "razoável", conforme observado na tabela 02.

Tabela 1 - Parâmetros de classificação do Teste Sentar e Alcançar (Banco de Wells), considerando o sexo e a idade

\begin{tabular}{lllllll}
\hline Idade & $40-49$ & & $50-59$ & & $60-69+$ & \\
Sexo & Masculino & Feminino & Masculino & Feminino & Masculino & Feminino \\
Excelente & $>35$ & $>38$ & $>35$ & $>39$ & $>33$ & $>35$ \\
Muito bom & $29-34$ & $34-37$ & $28-34$ & $33-38$ & $25-32$ & $31-34$ \\
Bom & $24-28$ & $30-33$ & $24-27$ & $30-32$ & $20-24$ & $27-30$ \\
Razoável & $18-23$ & $25-29$ & $16-23$ & $25-29$ & $15-19$ & $23-26$ \\
Necessita melhorar & $<17$ & $<24$ & $<15$ & $<24$ & $<14$ & $<22$
\end{tabular}

Fonte: Canadian Standardized Test of Fitness (CSTF) 
Tabela 2 : Dados relacionados ao teste Sentar e Alcançar pré e pós-tratamento

\begin{tabular}{llll}
\hline Paciente & Avaliação inicial & Reavaliação & \% de Melhora \\
1 & $19,33 \mathrm{~cm}\left({ }^{*} \mathrm{NM}\right)$ & $26 \mathrm{~cm}\left({ }^{*} \mathrm{R}\right)$ & $34,5 \%$ \\
2 & $26,33 \mathrm{~cm}\left({ }^{*} \mathrm{R}\right)$ & $25,6 \mathrm{~cm}\left({ }^{*} \mathrm{R}\right)$ & $-0,02 \%$ \\
3 & $21,33 \mathrm{~cm}\left({ }^{*} \mathrm{NM}\right)$ & $35,6 \mathrm{~cm}\left({ }^{*} \mathrm{E}\right)$ & $66,9 \%$ \\
4 & $0,6 \mathrm{~cm}\left({ }^{*} \mathrm{NM}\right)$ & $0,7 \mathrm{~cm}\left({ }^{*} \mathrm{NM}\right)$ & $1,6 \%$ \\
5 & $11,33 \mathrm{~cm}\left({ }^{*} \mathrm{NM}\right)$ & $18,3 \mathrm{~cm}\left({ }^{*} \mathrm{R}\right)$ & $61,5 \%$ \\
\hline
\end{tabular}

* $\mathrm{NM}=$ necessita melhorar, $\mathrm{R}=$ razoável, $\mathrm{E}=$ excelente

Ao final das 5 sessões, os pacientes foram reavaliados e demonstraram ganhos na flexibilidade do tronco e dos membros inferiores, destacando-se os pacientes 1,3 e 5, enquanto que o paciente 2 apresentou um pequeno retrocesso, podendo este estar relacionado ao quadro álgico na articulação glenoumeral na data da reavaliação.

Segundo Guadagnini et al.(2012), idosos participantes de programas de atividades físicas sistematizadas apresentam um nível de flexibilidade superior (70\%) à idosos que não realizam tais atividades (30\%), já que o sedentarismo gera o encurtamento de fibras musculares.

Em um estudo realizado por Delabary et al. (2016) onde se relacionou a prática da dança aliada aos aspectos flexibilidade e melhora da qualidade de vida, verificou-se que houve uma evolução significativa em ambas as variáveis estudadas. Estes dados são semelhantes aos analisados no respectivo estudo, onde foi constatado progresso tanto na qualidade de vida como também na flexibilidade dos idosos.

No referente ao equilíbrio, na avaliação inicial constatou-se que a maior parte da amostra se enquadrava com um risco de quedas de 6 a $8 \%$, com exceção de uma paciente que demonstrava um risco de quedas de $100 \%$. 
Ao observar os dados da tabela 3, ao fim da intervenção, nota-se que todos os pacientes apresentaram ganhos referentes ao equilíbrio, destacando-se a paciente 3 que apresentou uma melhora de 44,8\%, onde a mesma na avaliação inicial classificava-se na escala de Berg com o risco de quedas de $100 \%$ e após as sessões realizadas, obteve uma redução de 6 a $8 \%$.

Tabela 3: Escores relacionados à avaliação inicial e reavaliação dos pacientes através da Escala de Berg

\begin{tabular}{llll}
\hline Paciente & Avaliação inicial & Reavaliação & $\%$ de Melhora \\
1 & 43 pontos & 48 pontos & $11,6 \%$ \\
2 & 42 pontos & 47 pontos & $11,9 \%$ \\
3 & 29 pontos & 42 pontos & $44,8 \%$ \\
4 & 34 pontos & 44 pontos & $29,4 \%$ \\
5 & 43 pontos & 50 pontos & $16,2 \%$ \\
\hline
\end{tabular}

O envelhecimento compromete a habilidade do sistema nervoso central em efetuar o processamento dos sinais vestibulares, visuais e proprioceptivos responsáveis pela manutenção do equilíbrio corporal, bem como minimiza a capacidade de modificações dos reflexos adaptativos. Esses processos degenerativos são responsáveis pela ocorrência de desequilíbrio na população geriátrica (Ruwer et al., 2005).

A melhora do equilíbrio observada na presente pesquisa reafirma os resultados obtidos por Santana et al. (2008) que ao tratar 25 idosos utilizando a dançaterapia com duração de 60 minutos, verificaram melhora significativa neste quesito.

Quanto aos resultados obtidos pelo teste Time Up and Go, na avaliação inicial nota-se através da Tabela 04 que dois idosos em especial apresentavam um risco elevado de quedas e redução da agilidade, realizando o percurso com um tempo igual ou superior a 30 segundos. 
Tabela 4: Resultados iniciais e finais do teste de Agilidade Time Up and Go

\begin{tabular}{llll}
\hline Paciente & Avaliação inicial & Reavaliação & $\%$ de melhora \\
\hline 1 & 9.53 segundos & 7.76 segundos & $18,5 \%$ \\
2 & 14.16 segundos & 9.63 segundos & $31,9 \%$ \\
3 & 31.10 segundos & 21.26 segundos & $31,6 \%$ \\
4 & 30.65 segundos & 18.14 segundos & $40,8 \%$ \\
5 & 10.03 segundos & 7.55 segundos & $24,7 \%$ \\
\hline
\end{tabular}

Analisando a tabela 4, verifica-se que após as sessões de dançaterapia e cinesioterapia, houve melhora em todos os pacientes, sendo importante ressaltar a evolução do paciente número 4 , que ao final das sessões apresentou porcentagem de melhora de $40,8 \%$.

No referente à variável agilidade, os benefícios adquiridos com a dançaterapia no presente estudo são semelhantes aos obtidos por Barboza et al. (2013), onde ao tratar 61 idosos divididos em grupo experimental e controle utilizando a dança como modalidade terapêutica, verificaram diferença estatisticamente significante para o grupo experimental em relação ao grupo controle no tempo de realização do Time Up and Go .

Quanto ao questionário de qualidade de vida (SF-36) foi verificado aumento das médias nos escores obtidos em cada um dos domínios avaliados no pré e póstratamento, sendo importante destacar o item "limitação por aspectos emocionais", onde foi constatado um avanço importante na média final.

Tabela 5: Média e desvio padrão $( \pm S D)$ dos resultados da avaliação inicial do questionário de qualidade de vida

\begin{tabular}{llllll}
\hline Domínios do SF 36 & Média Inicial & $\begin{array}{l}\text { Desvio } \\
\text { inicial }\end{array}$ & Padrão & Média final & $\begin{array}{l}\text { Desvio padrão } \\
\text { final }\end{array}$ \\
$\begin{array}{l}\text { Capacidade } \\
\text { funcional }\end{array}$ & $75 \%$ & 20 & $94 \%$ & 10,8 & \\
$\begin{array}{l}\text { Limitação por } \\
\text { aspectos físicos }\end{array}$ & $55 \%$ & 51,2 & $85 \%$ & 33,5 \\
$\begin{array}{l}\text { Dor } \\
\text { Estado geral de }\end{array}$ & $32,8 \%$ & $30,4 \%$ & 11,9 & $62,2 \%$ & 17,4 \\
saúde & & & $39,6 \%$ & 21,9 \\
\end{tabular}




\begin{tabular}{lllll}
\hline Vitalidade & $54 \%$ & 13,8 & $89 \%$ & 16,3 \\
Aspectos sociais & $37,5 \%$ & 19,7 & $67,5 \%$ & 6,84 \\
Limitação por & $53,3 \%$ & 44,7 & $93,3 \%$ & 14,9 \\
$\begin{array}{l}\text { aspectos } \\
\text { emocionais }\end{array}$ & & & & \\
Saúde mental & $51,2 \%$ & 14,8 & $85,6 \%$ & 14,5 \\
\hline
\end{tabular}

Analisando pelo aspecto mental, a atividade física, principalmente quando realizada em grupo, contribui para a autoestima do idoso, favorece as relações psicossociais, o reequilíbrio emocional, além de também beneficiar o desempenho em tarefas seqüenciais, a capacidade de concentração e memória de curto prazo, sendo todos estes muito importantes para as funções cognitivas necessárias para a vida (Stella et al., 2002). Estas informações corroboram os resultados evidenciados em nosso estudo, já que os idosos obtiveram melhoras significativas em diversas variáveis do Teste SF-36.

São várias as modalidades de exercícios físicos voltados para a terceira idade, sendo que, dentre elas, a dança promove um aprimoramento da coordenação motora, da propriocepção, além de beneficiar o convívio social e o domínio psíquicos. Além disso, quando realizada com regularidade e duração de 30 a 60 minutos promove efeitos semelhantes aos de exercícios aeróbicos. Esta informação corrobora com os dados obtidos em nosso estudo, visto que foram observados ganho no equilíbrio, agilidade e qualidade de vida dos idosos tratados (Jacob, 2006).

\section{CONCLUSÃO}

O desenvolvimento do presente estudo possibilitou a conclusão de que a Dançaterapia aliada a Cinesioterapia promove efeitos benéficos e significativos na saúde da população idosa, em especial na flexibilidade, equilíbrio, agilidade e qualidade de vida, trazendo inúmeros benefícios para os indivíduos praticantes desta modalidade de atividade física.

\section{REFERÊNCIAS}


BARBOZA, Natália; FLORIANO, Eduardo; MOTTER, Bruna; SILVA, Flávia; SANTOS, Suhaila. Efetividade da Fisioterapia associada à dança em idosos saudáveis: ensaio clínico aleatório. Disponível em: <http://www.scielo.br/pdf/rbgg/v17n1/1809-9823-rbgg-17-01-00087.pdf>. Acesso em: $11 / 12 / 2018$.

CICONELLI, Rozana; FERRAZ, Marcos; SANTOS, Wilton; MEINÃO, Ivone; QUARESMA, Marina. Tradução para a língua portuguesa e validação do questionário genérico de avaliação de qualidade de vida SF-36 (Brasil SF-36). Revista Brasileira de Reumatologia, v.39, n.3, p. 143-150. mai./jun.1999.

DELABARY, Marcela; KOMEROSKI, Isabel; SCHUCH, Felipe; HAAS, Aline. Dança e flexibilidade: interferências na qualidade de vida de adultos. Revista Brasileira de Qualidade de Vida, v.8, n. 1, p. 16-27, jan./mar. 2016.

FITNESS CANADA. Canadian Standardized Test of Fitness (CSTF) Operations Manual. 3rd. Ed. Otawwa: Fitness and Amateur Sport, Canada, 1986.

GUADAGNINI, Pércio; OLIVOTO, Robson. Comparativo de flexibilidade em idosos praticantes e não praticantes de atividades físicas. EFDeportes.com/Revista Digital. Disponível em: <http://www.efdeportes.com/efd69/flexib.htm>. Acesso em $11 / 12 / 2018$.

JACOB, Wilson. Atividade física e envelhecimento saudável. Revista Brasileira Educação Física Esportiva, v. 20, n. 5, p. 73-77, set.2006.

KRAVCHYCHYN, Ana Cláudia; CAMACHO, Emanuella; BORGES, Paulo. O envelhecimento e suas implicações fisiológicas. EFDeportes.com/Revista Digital. Disponívelem:<http://www.efdeportes.com/efd169/o-envelhecimento-e-suasimplicacoes-fisiologicas.htm $>$. Acesso em: 11/12/2018.

LOPES KT, COSTA DF, SANTOS LF, CASTRO DP, BASTONE AC. Prevalência do medo de cair em uma população de idosos da comunidade e sua correlação com mobilidade, equilíbrio dinâmico, risco e histórico de quedas. Rev. BrasFisioter, v.13, n.3, p. 223-229, 03/02/2009.

MORAES, Edgar. Fisiologia do Envelhecimento aplicada à prática clínica. 1.ed.Belo Horizonte: Coopemed,2008.

NETTO, Francisco. Aspectos biológicos e fisiológicos do envelhecimento humano e suas implicações na saúde do idoso. Revista Pensar a Prática, v.7, n.1, p.75-84, mar. 2004.

ORGANIZAÇÃO MUNDIAL DE SAÚDE (OMS). Atividade física e saúde na Europa: Evidências para a acção. Centro de Investigação em Actividade Física, Saúde e Lazer. Disponívelem: $<$ https://ciafel.fade.up.pt/files download/actividade fisica e saude na europa.pdf>. Acessoem: 27/03/2018. 
PODSIADLO, Diane; RICHARDSON, Sandra. The timed "Up \& Go": a test of basic functional mobility for frail elderly persons. JAmGeriatrSoc, v.39, n.2, p.142-148, 1991.

REIS, Luciana; MASCARENHAS, Cláudio; LYRA, Joalise. Avaliação da qualidade de vida em idosos praticantes e não praticantes do método Pilates. C\&D - Revista Eletrônica da Fainor, v.4, n.1, p. 38-51, jan./dez. 2011.

RIBEIRO, Cibele; ABAD, Cesar; BARROS, Ronaldo; NETO, Turíbio. Nível de flexibilidade obtida pelo teste de sentar e alcançar a partir de estudo realizado na Grande São Paulo. Revista Brasileira de Cineantropometria\&Desempenho Humano, v.12, n.6, p.415-421, maio./jun. 2010.

RUWER, Sheelen; ROSSI, Angela; SIMON, Larissa. Equilíbrio no idoso. Revista brasileira de otorrinolaringologia, v. 71, n.3, p. 298-303, mai./jun. 2005.

SANTANA, Susana; CORRADINI, Amanda; CARNEIRO, Roberta. A dança de salão e seus benefícios motores, cognitivos e sociais. Anuário da Produção de Iniciação Científica Discente; v.12, n.15, p.83-104, 2009.

SCHEICHER, Marcos Eduardo; PIMENTEL, Renata. Comparação do risco de queda em idosos sedentários e ativos por meio da escala de equilíbrio de Berg. Revista Fisioterapia e Pesquisa, v.16, n.1, p.6-10, jan./mar. 2009.

SILVA, Andressa; ALMEIDA, Gustavo; CASSILHAS, Ricardo; COHEN, Moisés; PECCIN, Maria; TUFIK, Sérgio; MELLO, Marco. Equilíbrio, coordenação e agilidade dos idosos submetidos à prática de exercícios resistidos. Revista Brasileira de Medicina do Esporte, v. 14, n. 2, p. 88-93, mar./abr. 2008.

SPIRDUSO, Waneen. Dimensões físicas do envelhecimento. 1. ed. Barueri: Manole, 2005.

STELLA, Florindo; GOBBI, Sebastão; CORAZZA, Danilla; COSTA, José. Depressão no Idoso: Diagnóstico, Tratamento e Benefícios da Atividade Física. v.8, n.3, p. 91-98, ago./dez. 2002.

XAVIER, Geane; CAVALCANTI, Demétrius. Análise do nível de flexibilidade dos praticantes de treinamento resistido do centro de atividade física da cidade de Crato, CE.

EFDeportes.com,Revista

Digital.

Disponívelem:<https://www.efdeportes.com/efd181/analise-do-nivel-deflexibilidade.htm>. Acesso em: 11/12/2018. 